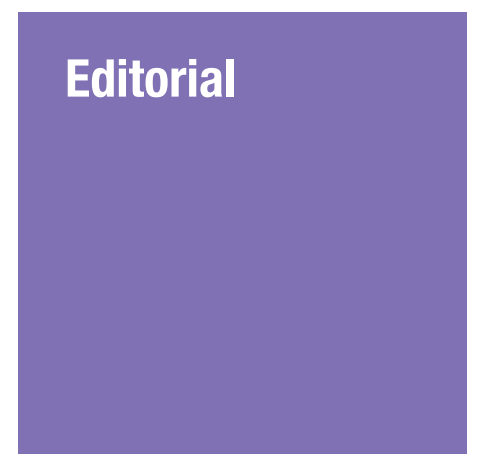

\title{
Progress in Clinical Neurosciences, Cognitive Neurosciences, Clinical Psychology, Neurotechnology and Brain Mapping in Malaysia
}

\author{
Jafri Malin AbduLLaH \\ Malaysian Journal of Medical Sciences, Universiti Sains Malaysia, Kubang \\ Kerian, Kelantan, Malaysia
}

Submitted: 1 Jan 2021

Accepted: 28 Feb 2021

Online: 21 Apr 2021

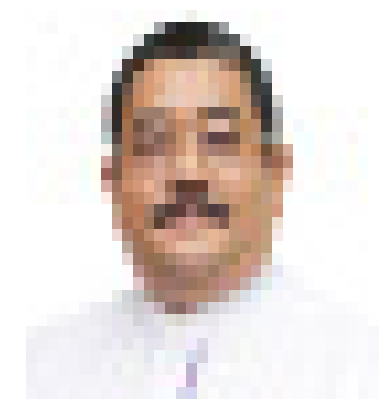

To cite this article: Abdullah JM. Progress in clinical neurosciences, cognitive neurosciences, clinical psychology, neurotechnology and brain mapping in Malaysia. Malays J Med Sci. 2021;28(2):1-14. https://doi.org/10.21315/ mjms2021.28.2.1

To link to this article: https://doi.org/10.21315/mjms2021.28.2.1

\begin{abstract}
Last year, there was an increase in the amount of manpower in Malaysia, especially in terms of the numbers of neurosurgeons, cognitive neuroscientists and clinical psychologists. One way to increase the number of cognitive neurotechnologists in the country in 2021 is to allow neuroscientists to register as neurotechnologists with the Malaysian Board of Technologists (MBOT). The Malaysian Brain Mapping project has risen from its humble beginnings as an initiative of the Universiti Sains Malaysia Brain Mapping Group in 2017. There is currently a proposal for its entry into the national arena via the Precision Medicine Initiative with the Academy Science Malaysia, the Ministry of Science, Technology and Innovation, Ministry of Higher Education and Ministry of Health. The current Malaysian Government's Science, Technology, Innovation and Economy (STIE) plan was launched in 2020, leading to the establishment of neurotechnology as one of 10 STIE drivers.
\end{abstract}

Keywords: neurotechnology, brain mapping, manpower, clinical psychologist, cognitive neuroscientist, neurosciences, precision medicine, Malaysia 


\section{Introduction}

On 14 July 2020, the Prime Minister of Malaysia, Tan Sri Muhyiddin Yassin, chaired the National Science Council with the Ministry of Science, Technology and Innovation's Minister Khairy Jamaluddin, Deputy Minister Ahmad Amzad Hasim and Secretary-General Datuk Ir Dr Siti Hamisah Tapsir and launched 10 Science, Technology, Innovation and Economy (STIE) drivers in accordance with the Malaysian National Policy on Science, Technology and Innovation (DSTIN) 2021-2030. One of the STIE drivers was neurotechnology (1).

Neurotechnology took many years to be recognised in Malaysia. In 2017, the Academy of Science Malaysia prepared a report entitled Science \& Technology Foresight Malaysia 2050: Emerging Science, Engineering \& Technology (ESET) Study. Then, in December 2020, the 1010 MySTIE framework, which trailblazed a path for prosperity, societal well-being and global competitiveness, was published and officiated by Minister Jamaluddin with the New Science Policy: DSTIN $2030(2,3)$.

On 15 December 2020, Bank Negara Malaysia established a RM1 billion High Tech Facility-National Investment Aspiration (HTFNIA) as part of its efforts to provide additional assistance for small medium enterprises (SMEs) affected by COVID-19. SME project participants in key government programmes involved in research, development and innovation for critical technologies identified under national blueprints from IR 4.0-related technologies, green technology and biotechnology to ensure continuity and the completion of existing projects. These technologies included blockchain, artificial intelligence, big data analytics, internet of things, addictive manufacturing $(3 \mathrm{D} / 4 \mathrm{D} / 5 \mathrm{D} / 6 \mathrm{D}$ printing), cybersecurity, system integrators, augmented reality, advanced materials, drones and manufacturing systems as well as bioscience technology and neurotechnology (4).

\section{Moving a New Generation Forward During the COVID-19 Pandemic in Malaysia}

Figures 1-5 show the current batches of manpower being trained after our last report a year ago $(5,6)$. The percentages of our neuroscience and psychology graduates being hired up to 2021 from the end of 2019 were: $100 \%$ for Masters of Surgery (Neurosurgery) and Advanced Masters of Medicine (Neurology), 50\% for PhDs/Doctorate, $55 \%-75 \%$ for the Masters of Cognitive Neurosciences and the Integrated Programme, and 44\% for the Clinical Psychology graduates (7).

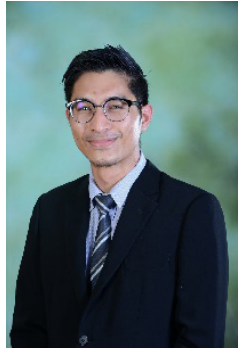

DR ABDUL HALEEM BIN NOORSHAM

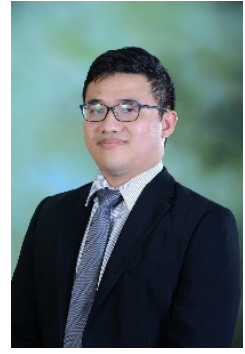

DR AIMAN ASYRAF BIN AHMAD SUKARI

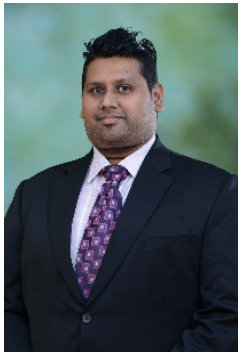

DR

JAGATHESAN SATHIVELOO

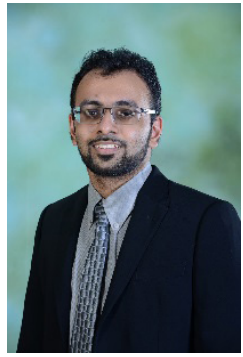

DR JONATHAN JOSEPH J NAESARAJOO

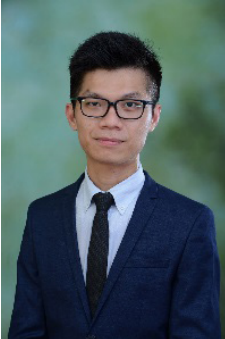

DR LEONARD

LEONG SANG XIAN

Figure 1. (continued on next page) 
Editorial | Neurotechnology STIE and human resources Malaysia

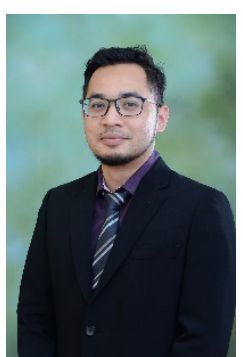

DR MOHAMAD MUHAIMIN BIN

ABDULLAH

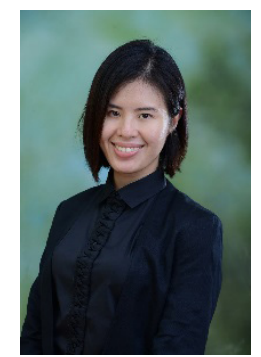

DR OOI LIN-WEI

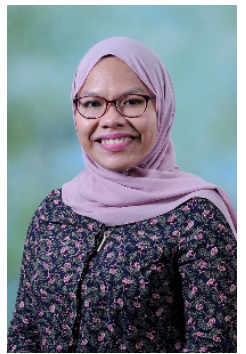

DR ANIS

NABILLAH MOHD AZLI

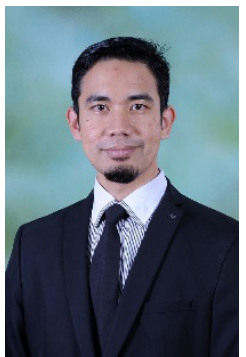

DR MOHAMMAD IMRAN BIN AHMAD

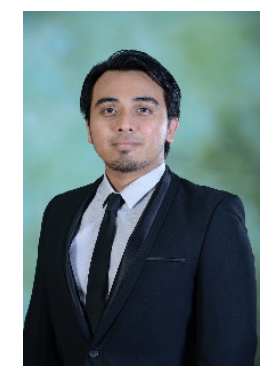

DR MUHAMMAD HAFIZ BIN HAJI MOHAMAD BOHARI

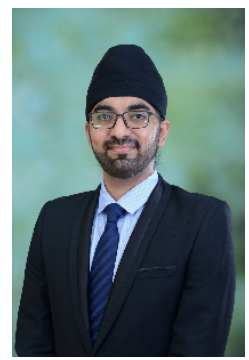

DR SARWINDER SINGH BHARMJIT SINGH

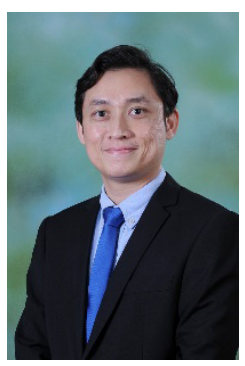

DR KHAIRUL AIZAD BIN ADZMAN

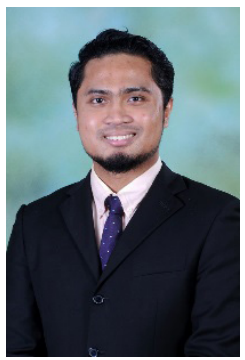

DR MOHAMMAD ISKANDAR BIN SA'UAD

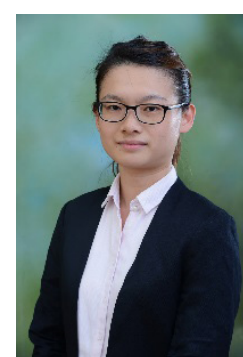

DR NG PEI MENG

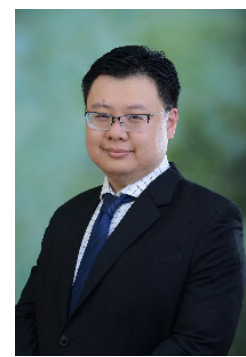

DR YONG DE JUN

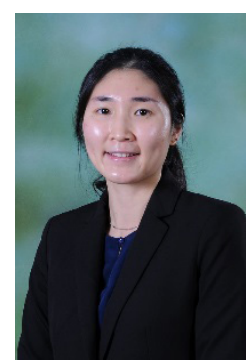

DR KHOO YEE HWA

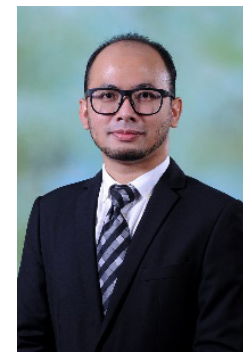

DR MOHD ARMAN BIN MUHAMAD NOR

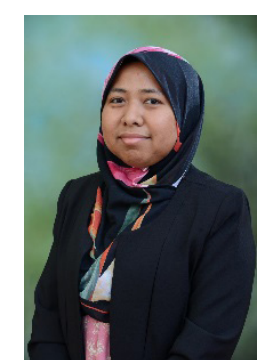

DR NOR BAIZURA BINTI ISMAIL

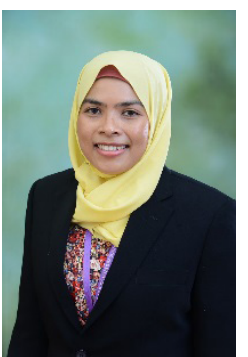

DR ZAITUN ZAKARIA

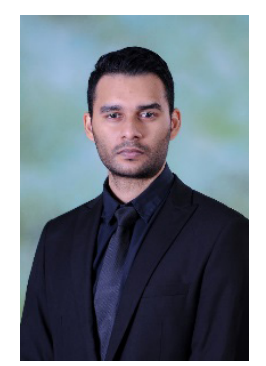

DR KUGAN VIJIAN

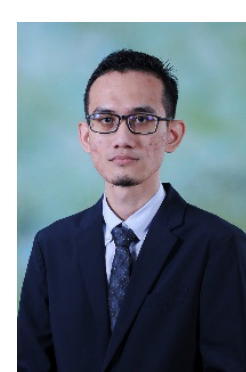

DR MUHAMMAD ADAM BIN ZAINUDDIN

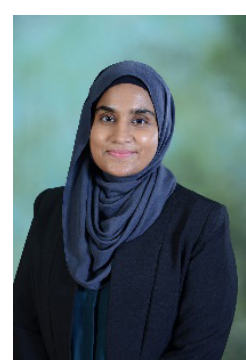

DR NUR

NAZLEEN BINTI SAID MOGUTHAM

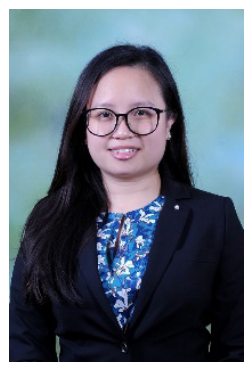

DR ALVERNIA

NEYSA BINTI UJAT

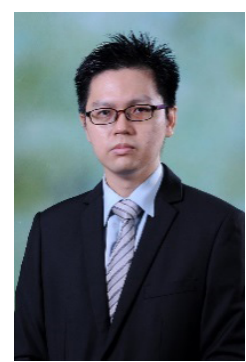

DR LEE KING PENG

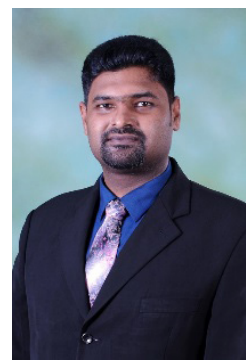

DR NAAVIN KUMAR BALAKRISHNAAN

Figure 1. (continued on next page) 


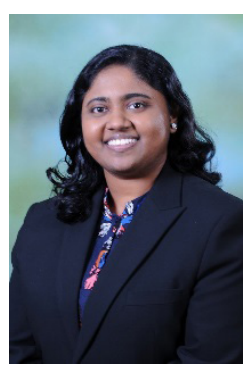

DR NISHANTHI APPAROW

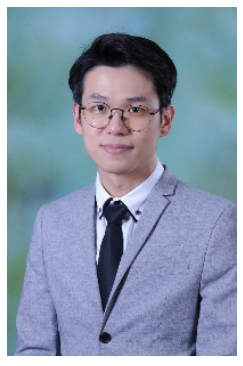

DR YAP TECK CHENG

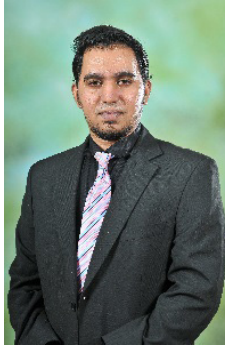

DR HARVINTH NAGALINGAM MUNIANDY

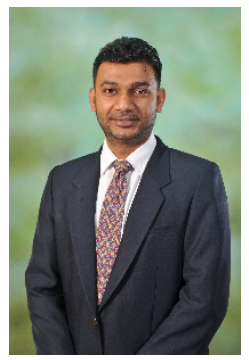

DR KUHA RAJ A/L ARUMUGAM

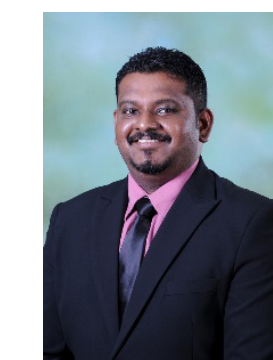

DR SAKTI VINAYAGA TAMIL SALVAN

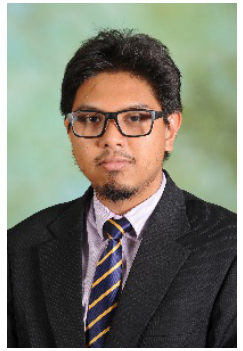

DR AHMAD ZULFADLI BIN MOHAMED RADZI

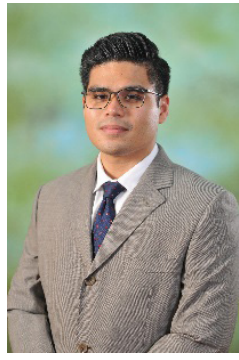

DR HEZRY ABU HASAN

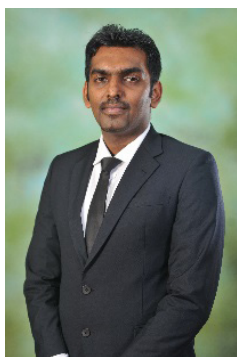

DR KUMARAPPAN A/L CHOCKALINGAM

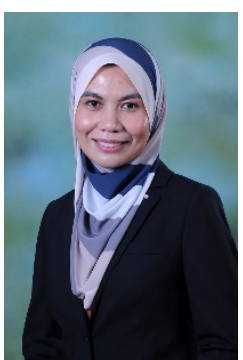

DR SHARIFAH NAWAL BINTI SYED JAAFAR

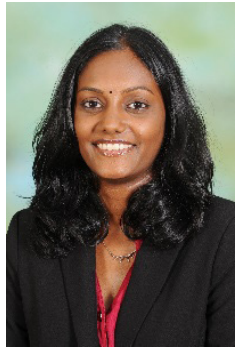

DR ALARMELU NITHYA A/P RAMANATHAN

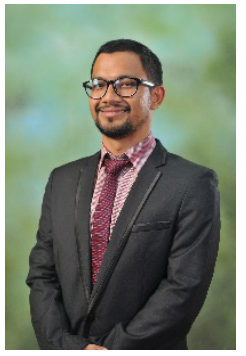

DR IDRIS SHAHROM

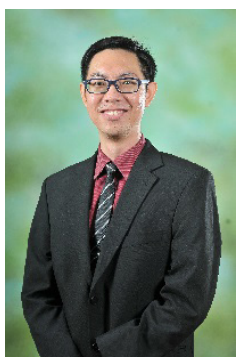

DR LOOI MUN CHOON

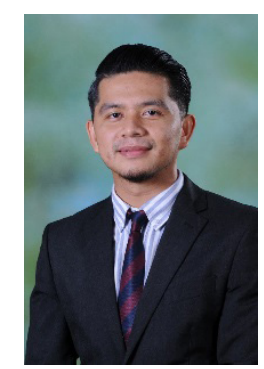

DR

SUZUANHAFIZAN BIN OMAR

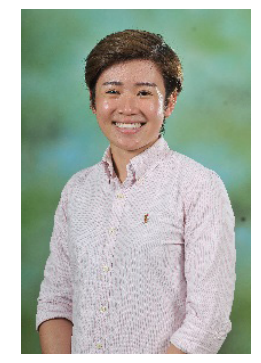

DR DEBBIE KONG CHING CHING

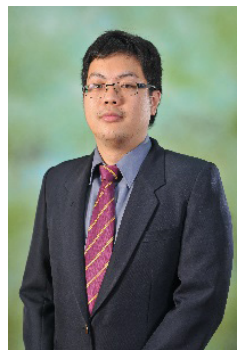

DR JESSE ZEN NGUI

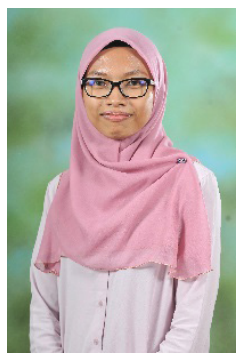

DR MAS SYAZANEEZA BINTI SHAB

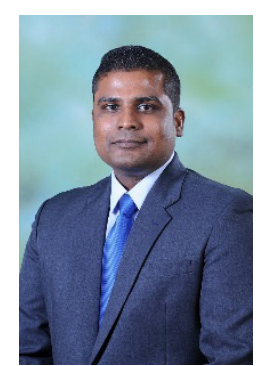

DR UTHAYA KUMAR NALLAYAN

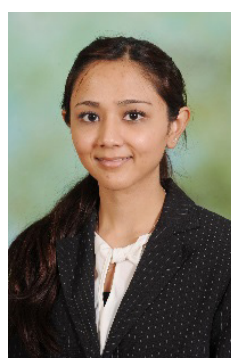

DR DIANA NOMA BINTI FITZROL

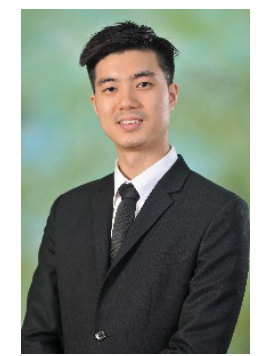

DR JULIAN TAN LI KWANG

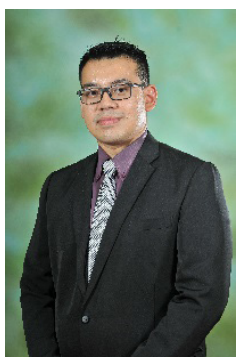

DR MOHD FARHAN BIN MOHD FAIZ WILSON YEO

Figure 1. (continued on next page) 
Editorial | Neurotechnology STIE and human resources Malaysia

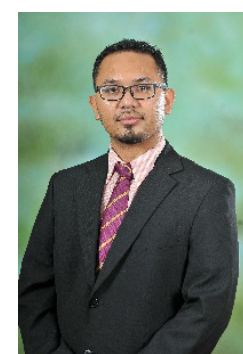

DR MOHD

GHADAFI BIN

WAHAB

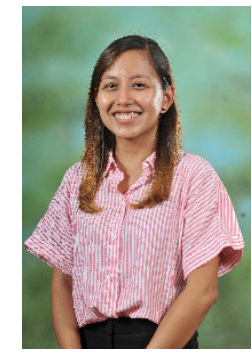

DR NADIAH BINTI AHMAD FUAD

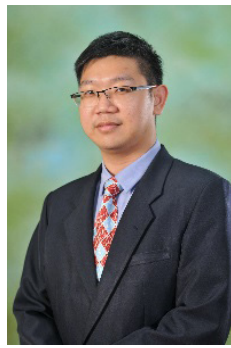

DR SAM JO EE

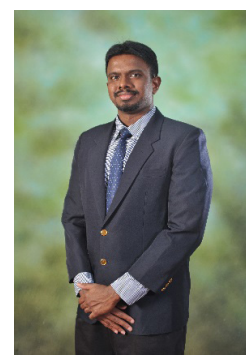

DR THAVANESAN

A/L

S.PUVANEVARAN

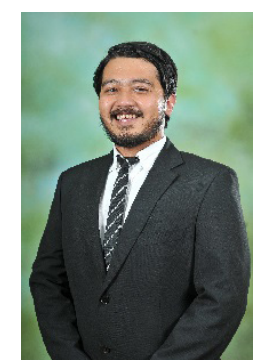

DR MOHD IRYAN BIN

CHE OTHMAN

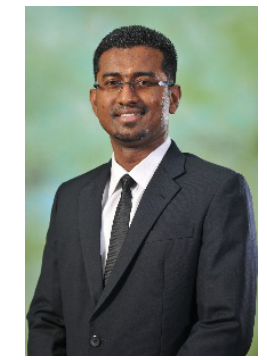

DR NISHAN RAO A/L SUBRAMANIAM

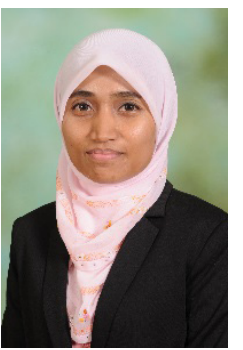

DR SARAH 'ATIQAH BINTI MOHD ZAMRI

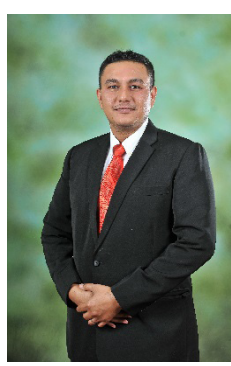

DR V JEYASEELAN G VASANTHAKUMARAN

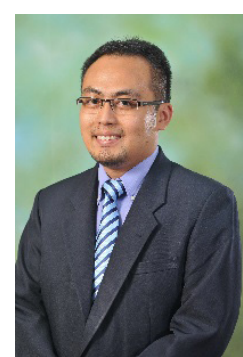

DR MOHD

KHAIRUN MOHD MISPAN

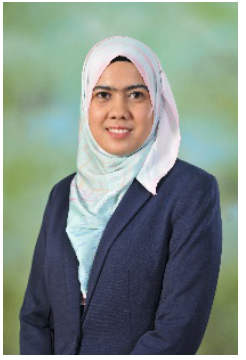

DR

NURSHAHEDA

BINTI MOHD

SALLEH

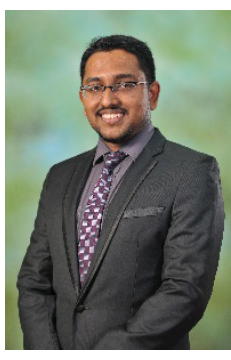

DR SARAVANAN A/L SRIDHARAN

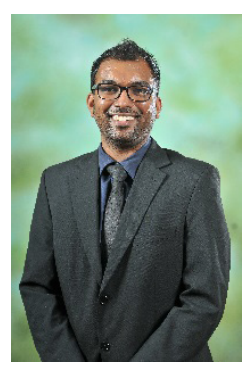

DR VICNESH THILLYNATHAN

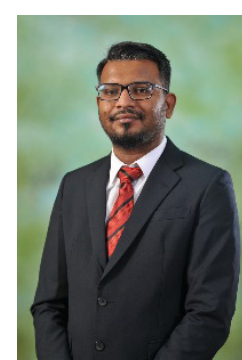

DR

MOVENTHIRAN

$\mathrm{A} / \mathrm{L}$

RAMAKRISHNAN

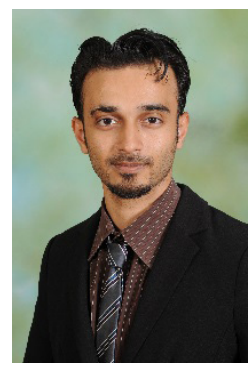

DR RAZMEENDER SINGH KELLY

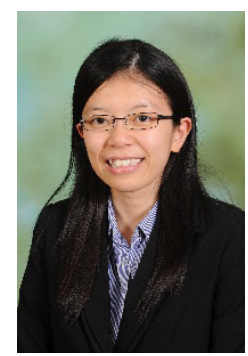

DR TAN SHZE EE

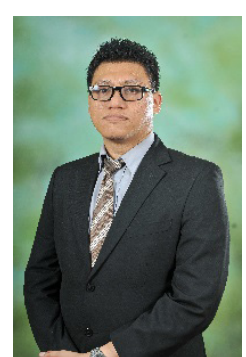

DR ZAHARUL AZRAN BIN ZAHARI

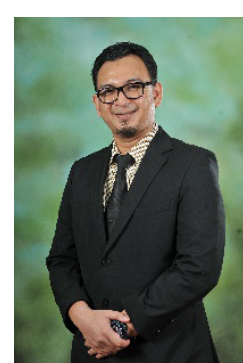

DR MUHAMAD RIDZUAN BIN ALIAS

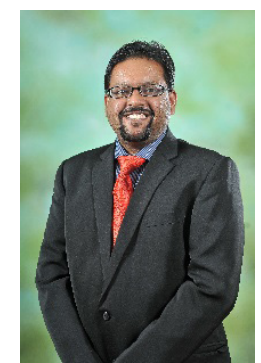

DR ROHAN JEEVARAJ

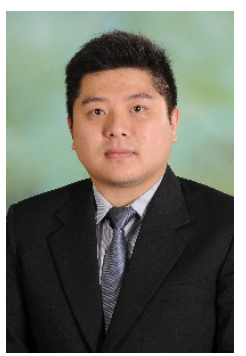

DR TAN ZI HAN

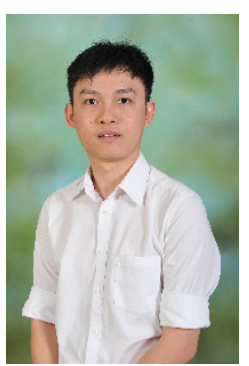

DR ANG SONG

YEE

Figure 1. (continued on next page) 


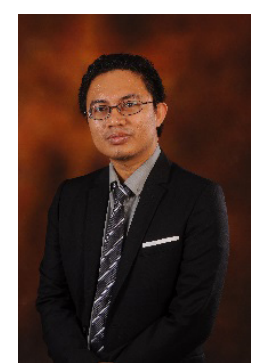

DR MUHAMMAD

NAJMI ABDUL

HALIM

Figure 1. Masters of Surgery (Neurosurgery) residents from May 2019 till December 2020

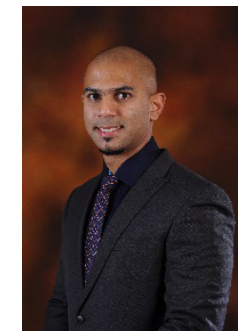

DR DAVENDRAN A/L KANESEN

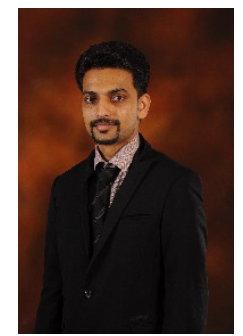

DR RAMISSH

PARAMASIVAM

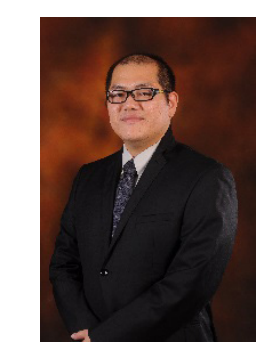

DR LAU BIK LIANG

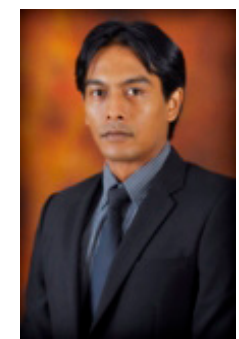

DR RAKESH RETHINASAMY

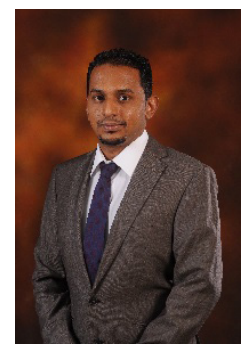

DR VINODH

VAYARA

PERUMALL

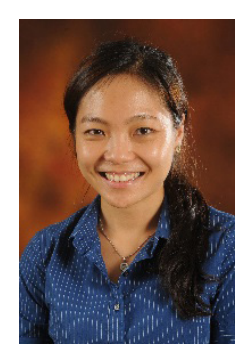

DR LEE CHUN LIN

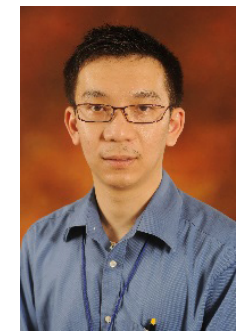

DR KHO GIAT SENG

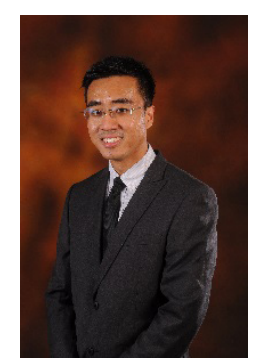

DR YEE SZE-VOON

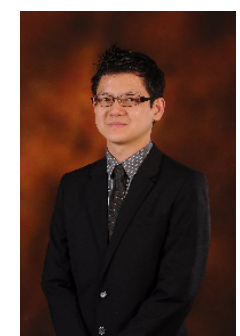

DR NELSON YAP KOK BING

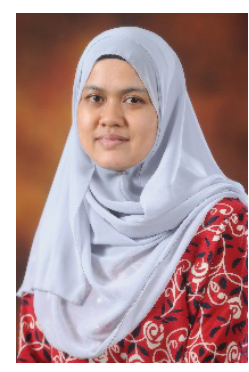

DR ASMA MUHAMAD AFIFI

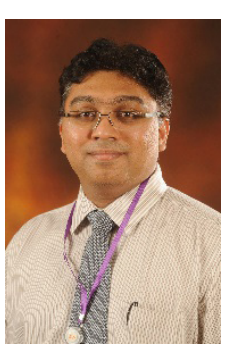

DR RAMANI THIAGARAJAH

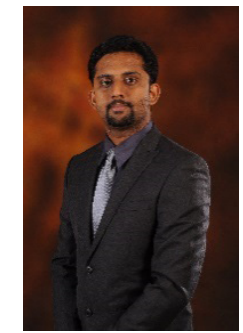

DR RAJENDRA RAO RAMALU

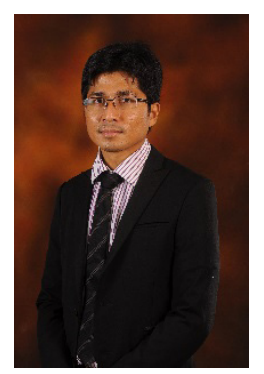

DR ASRARUL FIKRI ABU HASSAN

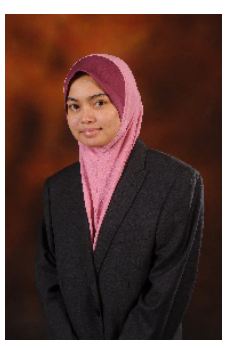

DR SHUKRIYAH SULONG

Figure 2. (continued on next page) 
Editorial | Neurotechnology STIE and human resources Malaysia

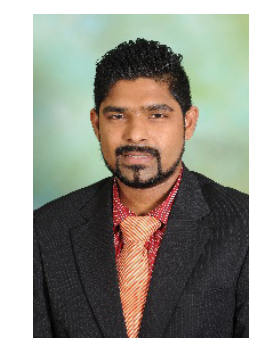

DR ARULKANESH DEVATATHAN

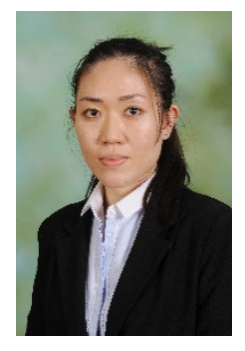

DR LIM MEI SIN

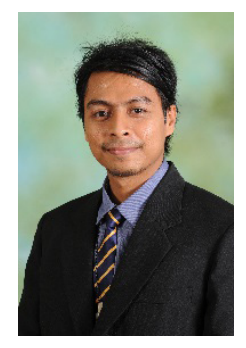

DR MUHAMMAD AIZZAT BIN OTHMAN

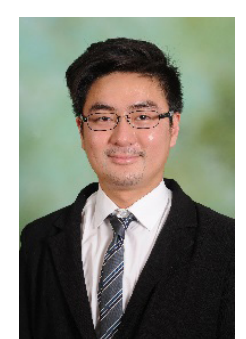

DR TEO EU GENE

Figure 2. Neurosurgeons who have graduated from postgraduate neurosurgical programme from 2019

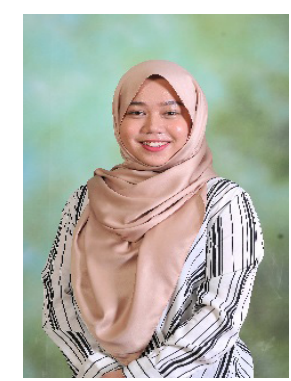

MSc - FATEN ANIS SYAIRAH SERI

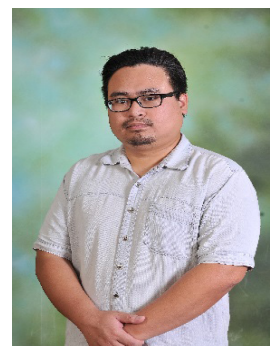

PhD - MUHAMAD RIDHA ABDUL RAHMAN

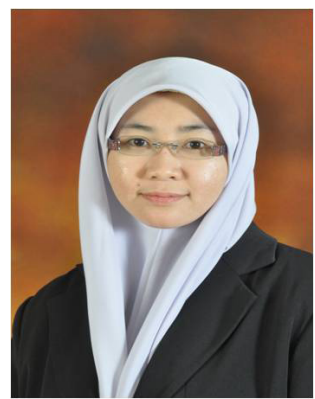

$\mathrm{PhD}$ - SAMHANI ISMAIL

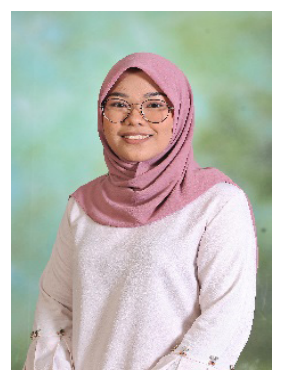

MSc - NIK NUR AZHANI ANUAR

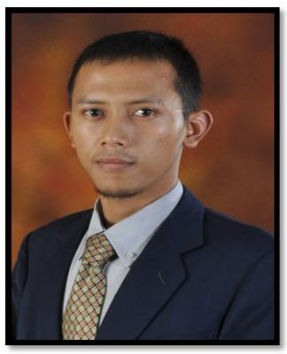

PhD -

MUHAMMAD HANIF CHE LAH

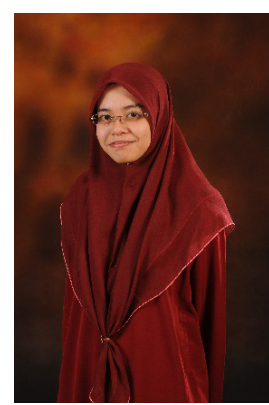

$\mathrm{PhD}$ - SITI MUSLIHAH ABD

RADZAK

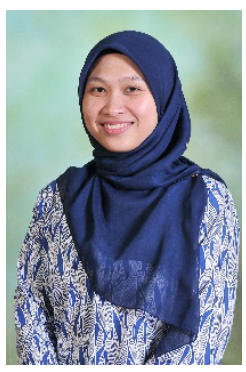

MSc - SITI NAZIHAHASMA HASSAN

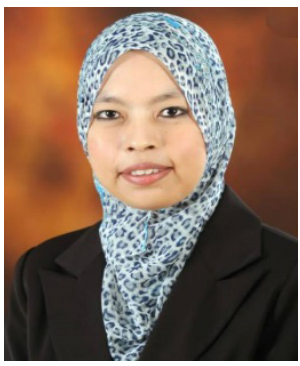

PhD - NOR SAFIRA ELAINA MOHD NOOR

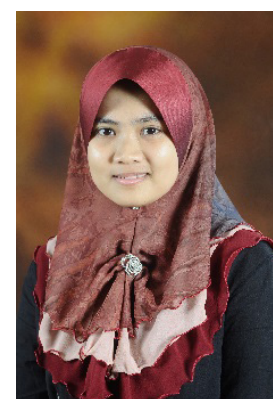

PhD - SITI

ZULAIKHA NASHWA MOHD KHAIR

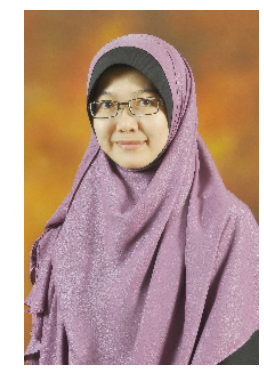

PhD - PUTRI NUR HIDAYAH AL ZIKRI MOHAMAD AKIL

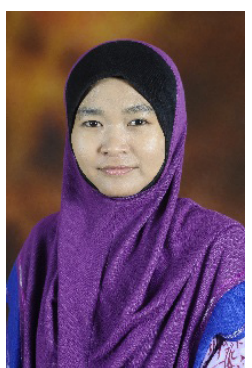

PhD - NUR SYAIRAH AB RANI

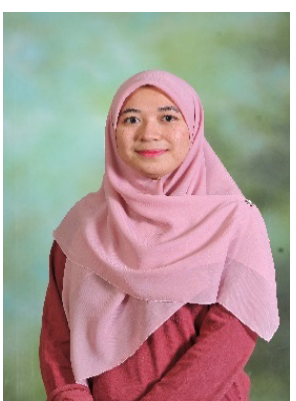

PhD - WAN NOR ADIBAH WAN ZAKARIA

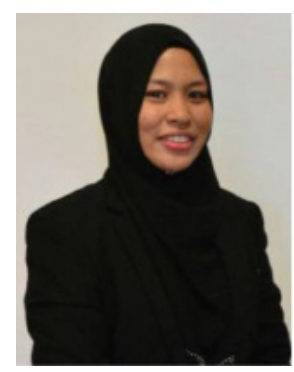

PhD - FATIN HANIZA ZAKARIA

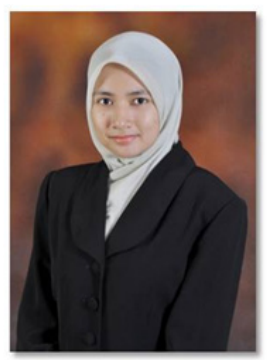

PhD - NURUL

IMAN WAN ISMAIL

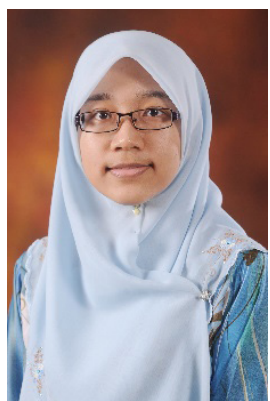

PhD - FATIN

HILYANI

MOHAMAD

Figure 3. (continued on next page) 


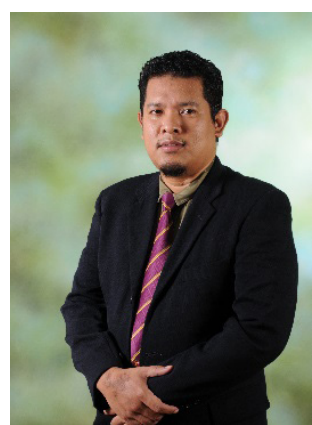

PhD - HAZIM OMAR

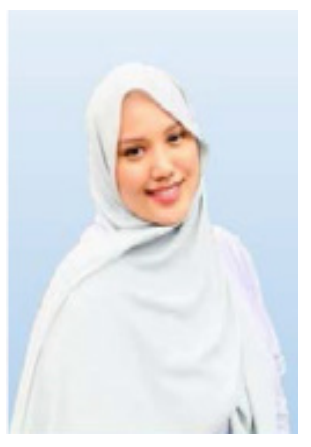

INP - NUR

SYAZRENA

ASHYQEEN

MUSTAFFA

KAMAL

Figure 3.Masters by Pure Research/Mixed Mode and PhD by Pure Research from May 2019

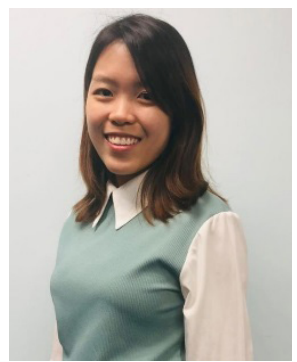

CHANG KAI RU

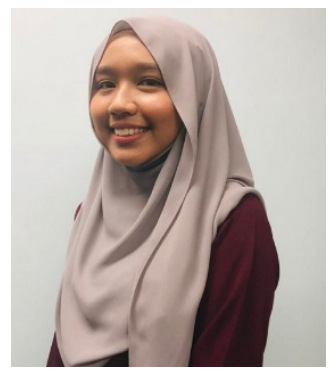

SYARAFANA HAZIRAH ZULKIFLI

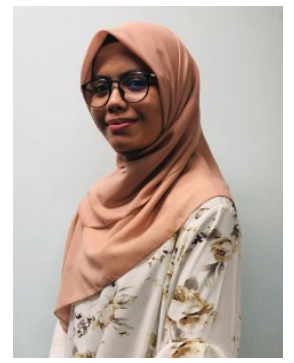

NUR SYAFIQAH SYAHRAIN MOHD SHAFAWI

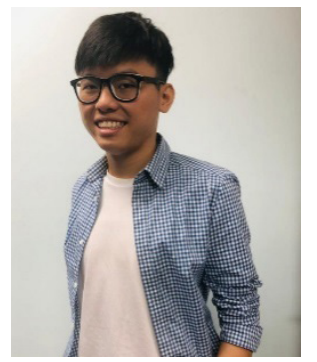

AW JIAN XIN

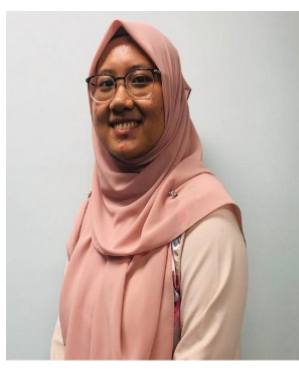

LIYANA BINTI MOHD TARMIZI

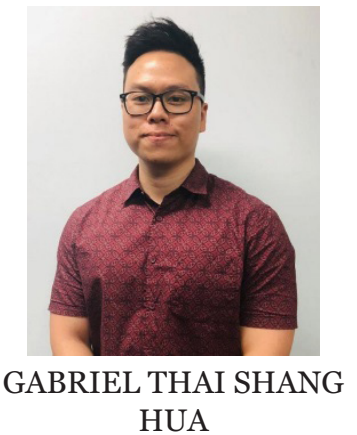

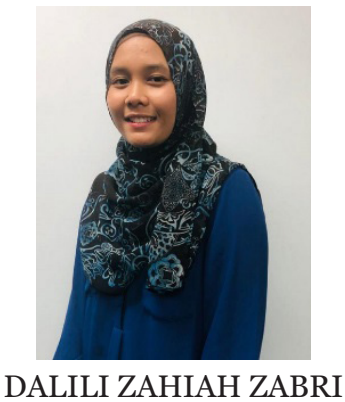

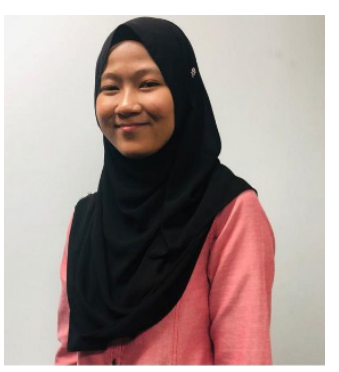

NUR SYAHRAIN KAMARULZAMAN

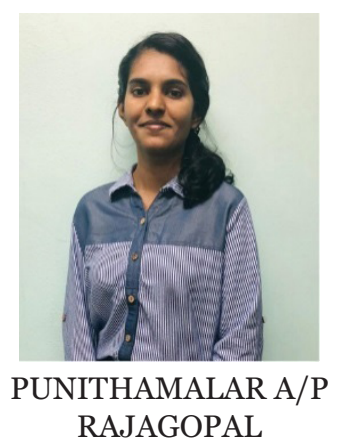

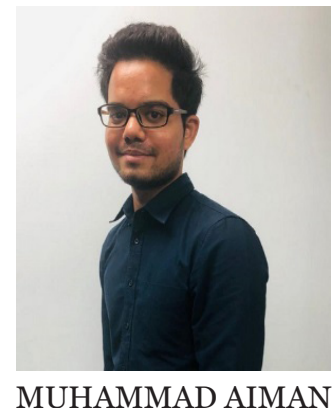
ISMAIL

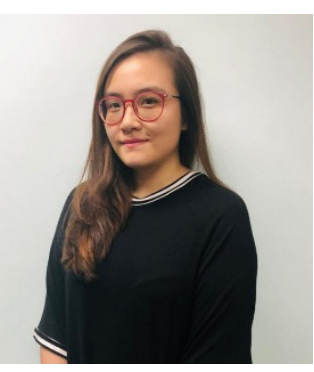

AFFAF NOOR SAIDI

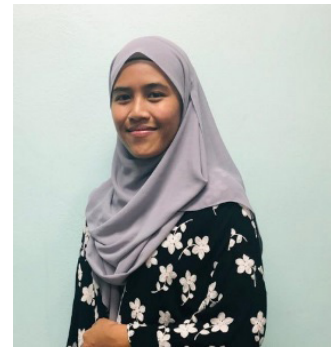

FATIN NURAFIQAH

Figure 4. (continued on next page) 
Editorial | Neurotechnology STIE and human resources Malaysia

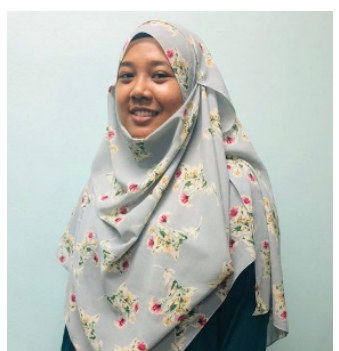

NURAIN MD YUSRI

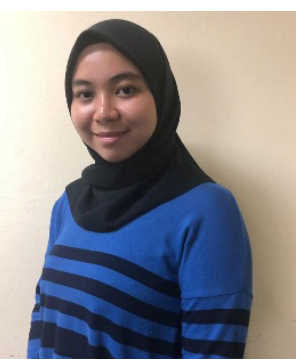

HAZIRAH ZULKIFLI

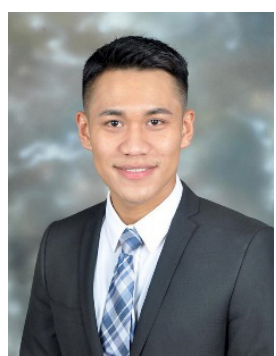

ALI SAMAT

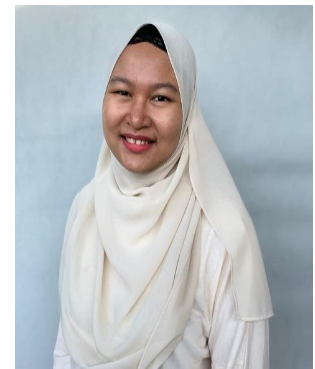

AUNEI ANUAR

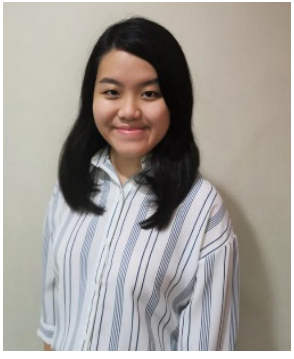

CHONG SHAO YIN

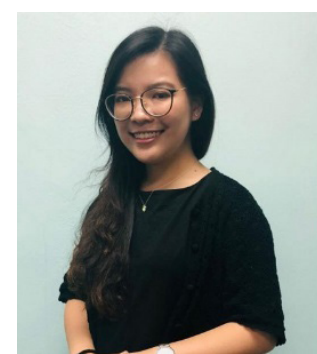

OON YEN MIN

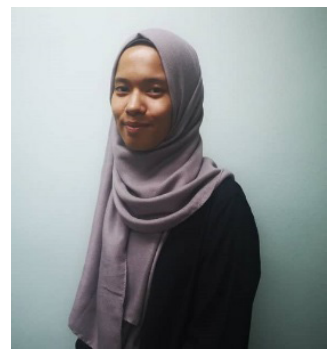

NUR HANI LIYANA KAMARUL AZHAR

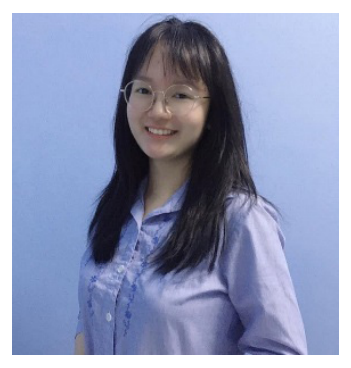

ALICIA NG CHER CHING

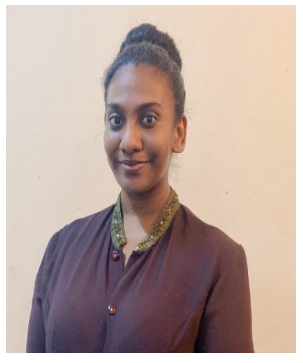

BANU JOTHIMALARR

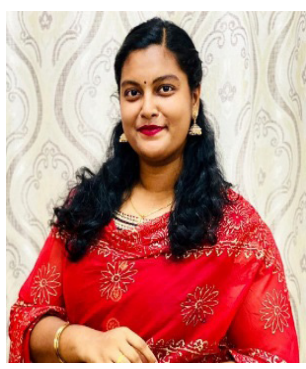

DITHIAMALAR LENGATHARAN

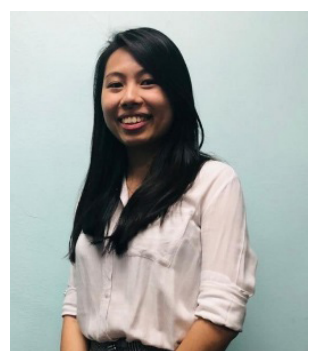

TAN JING HON

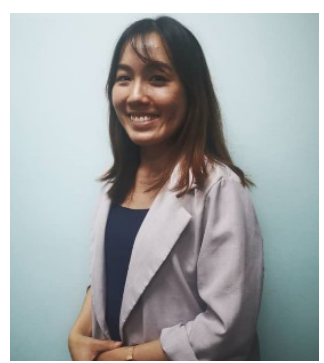

EVELYN NGUI AILING

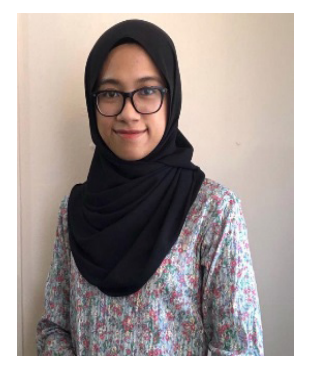

AMIRAH ZULAIKHA ZAFRUL AZLAN

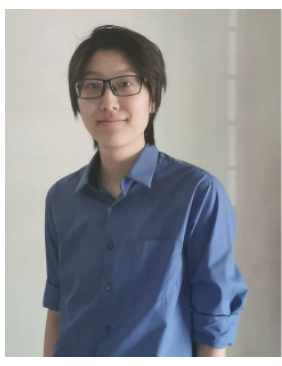

CHAN KAH MUN

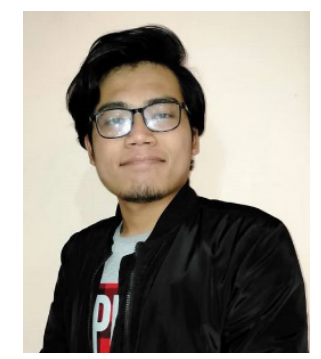

FAKHARUDIN AIMAN JAMAL

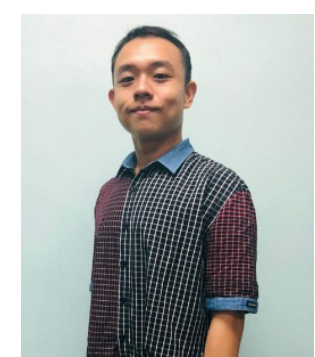

LIM CHEAN WEI

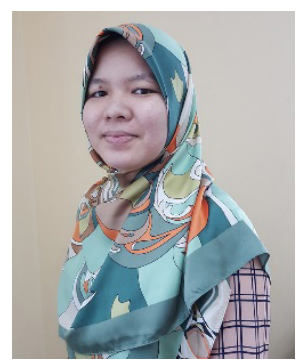

AMIRAH MUDRIKAH AMIRRUDDIN

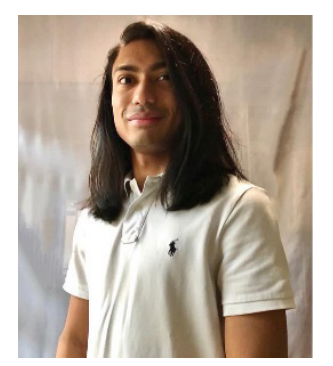

ARMAN IMRAN ASHOK

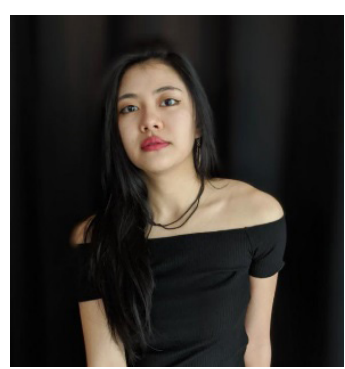

CHIN YENG ZIEN

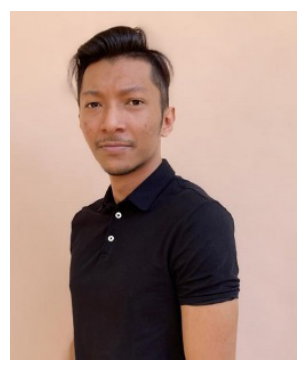

KHAIR BENJAMIN LOKMAN

Figure 4. (continued on next page) 


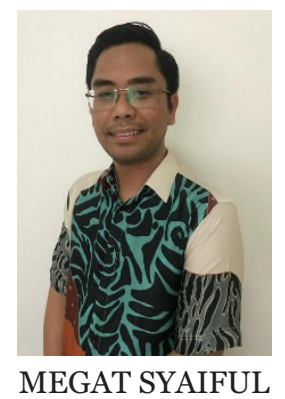

IZZUDDIN MEGAT

MOKHTAR

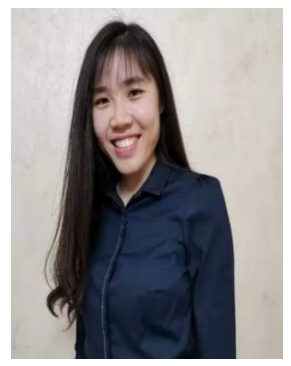

PHOON JU YEE

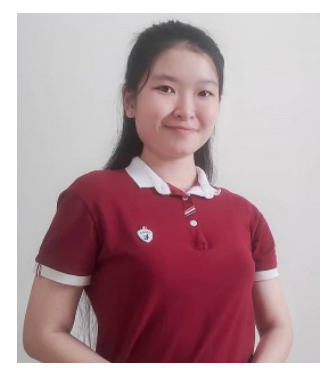

TAN JIUN TING

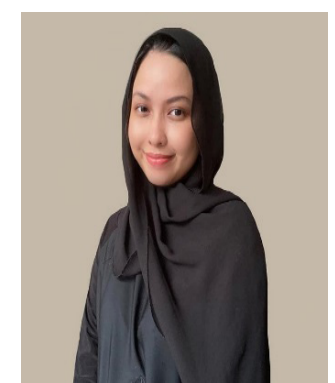

WAN FARAH ADILAH WAN AZLAN

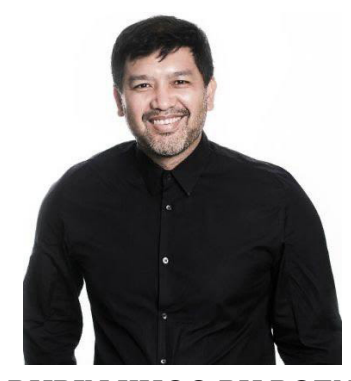

RUBIN KHOO BU BOEN

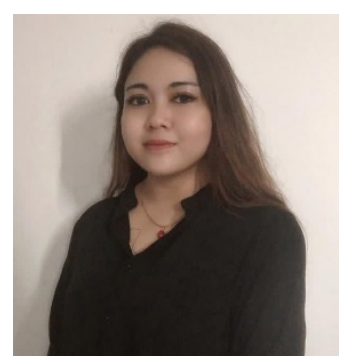

WAN ADIBAH NADIAH ABD RAZAK

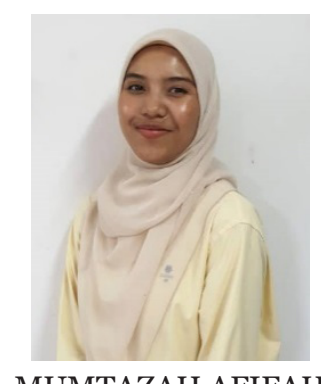

MUMTAZAH AFIFAH ABDUL HALIM

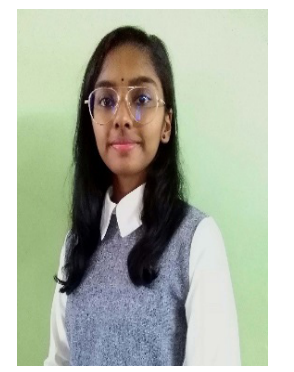

SHAMNI MARKUNDU

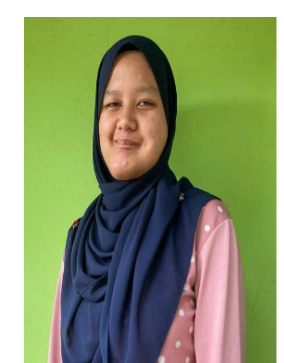

NUR HAFIZAH ZAINOL
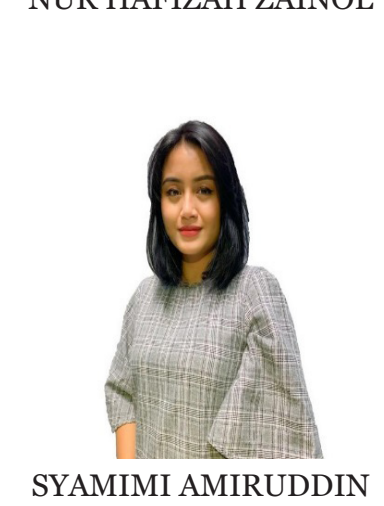

Figure 4. Second and third batch of Clinical Psychology students USM-UPSI

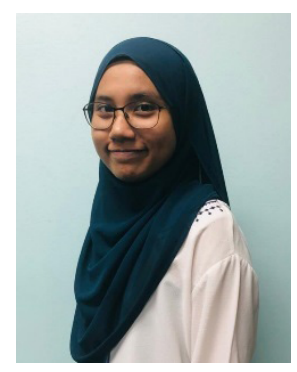

AMIRA MAISARAH MOHD DAUD

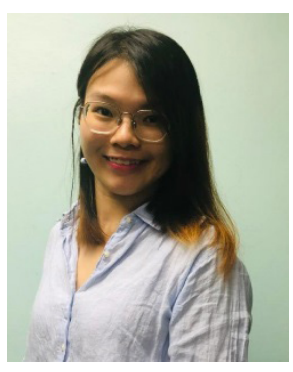

CHANG SHU CHUAN

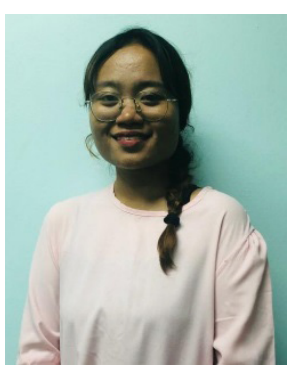

HUSNA MD ISA

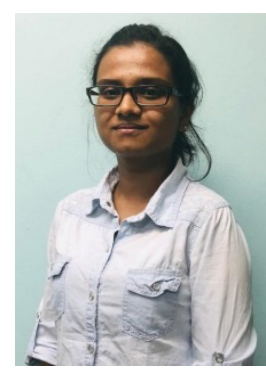

INTHU JAA A/P GOVINDAN

Figure 5. (continued on next page) 
Editorial | Neurotechnology STIE and human resources Malaysia

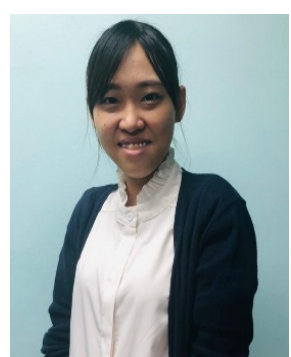

KONG YEE MUN

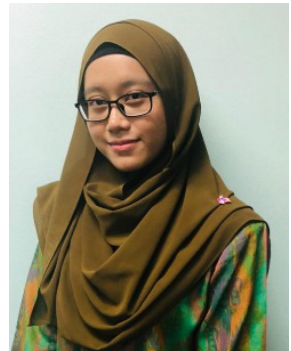

MARYAM ADAM

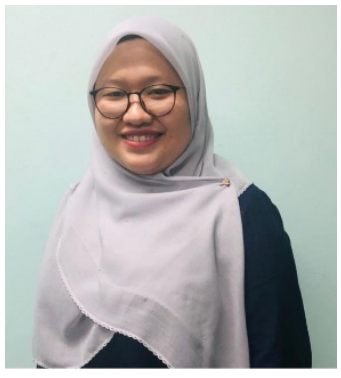

NUR FAHIMAH AHMAD SANDARA LELA PUTERA

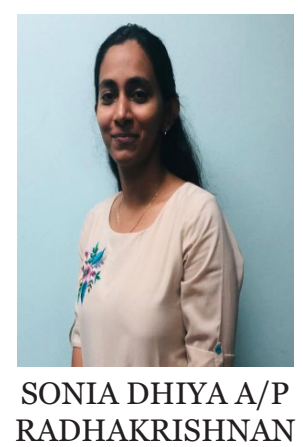

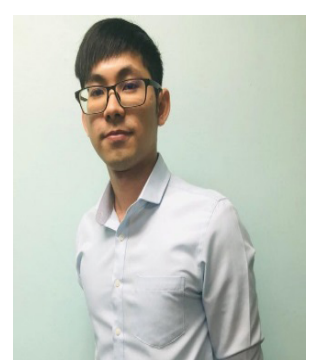

KOW WEN XUAN

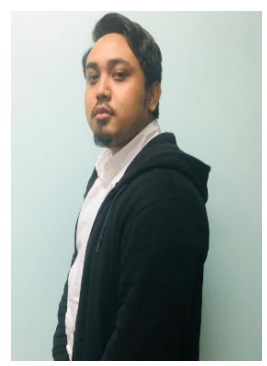

MUHAMMAD NASIRRUDDIN TAIJADIN

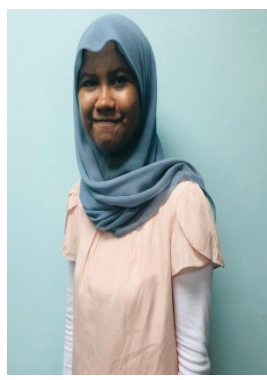

NURSABRINA MOHD FIRDAUS ALOYSIOUS

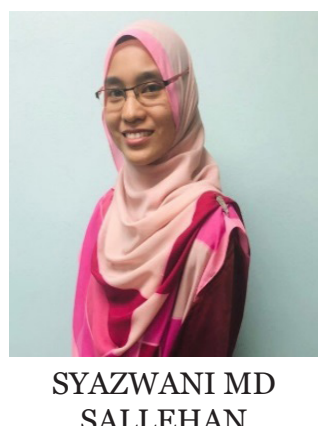

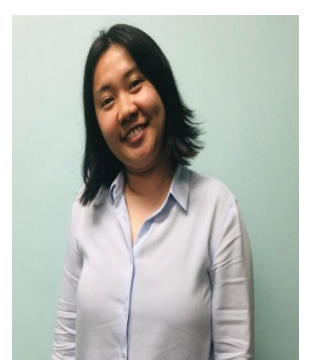

LEE HUEY YI

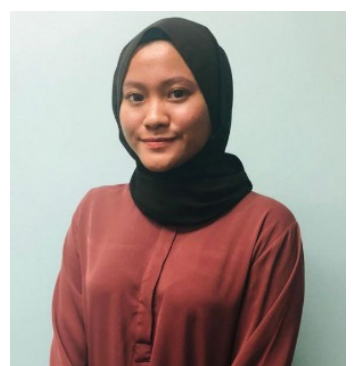

NADHIRAH KHAIDZIR

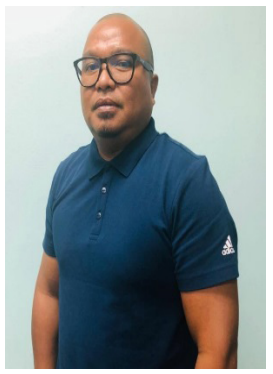

SHAMSUL OMAR TAJIDIN

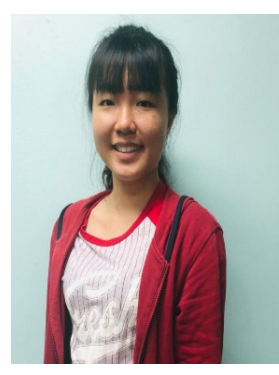

TAN WEI TING

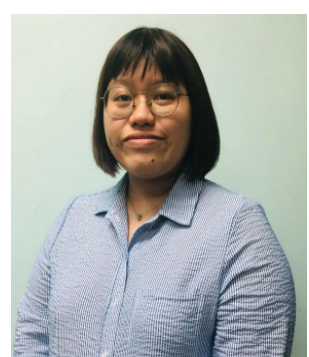

LEE JA NICE
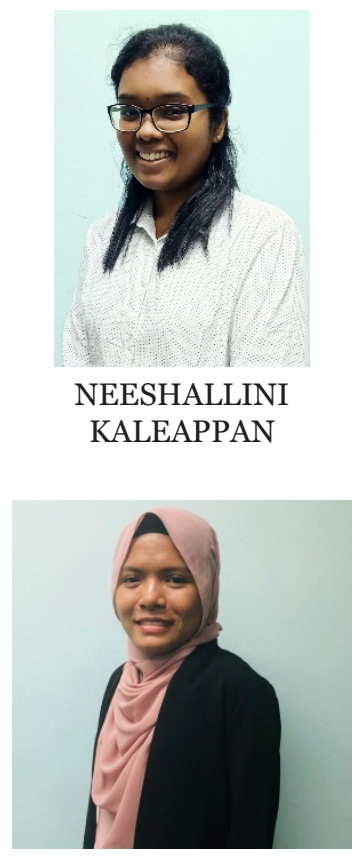

SITI HAJAR ZABRI

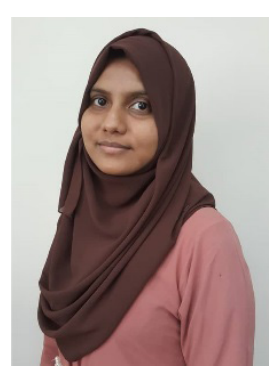

UNAISA SAUD

Figure 5. (continued on next page) 


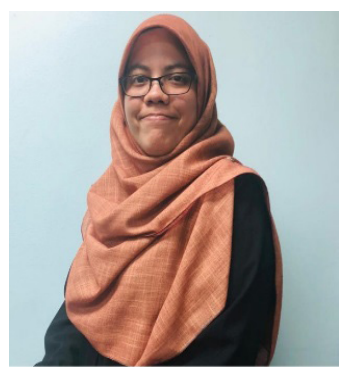

WAN SARAH WAN AHMAD KAMIL

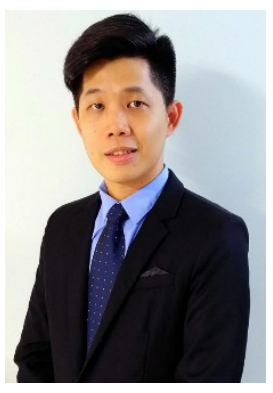

CHIN CHUN MING
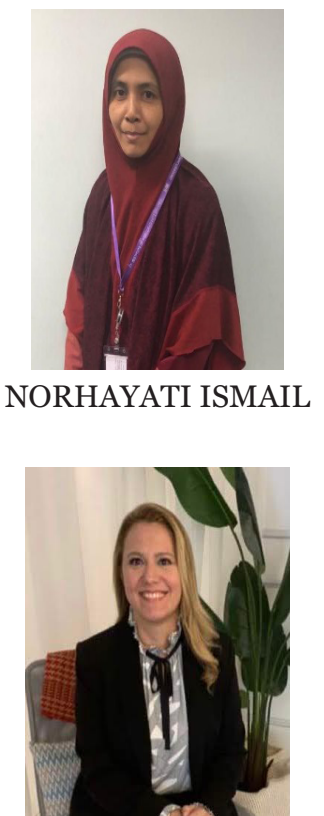

ELIF MALMQVIST

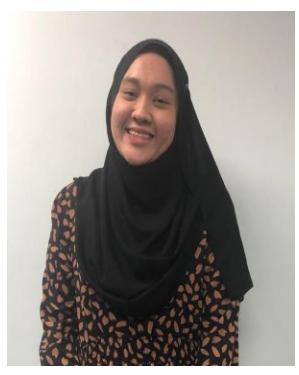

ANNIS SHAFIKA AMRAN
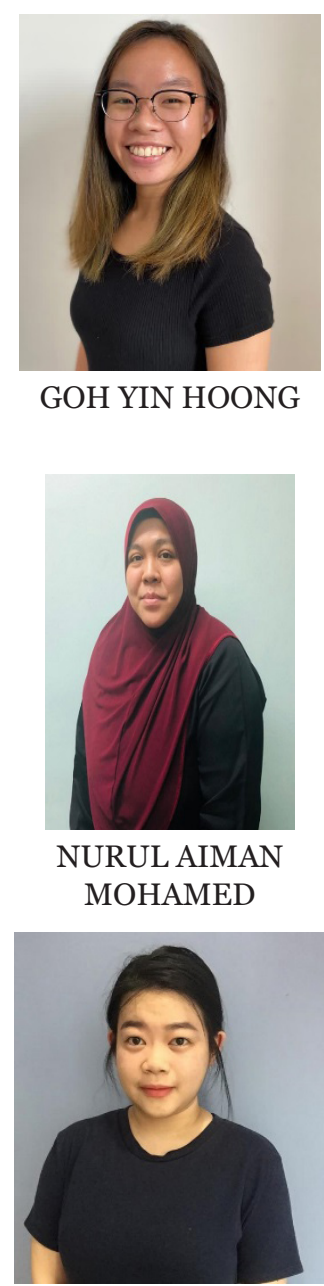

FONG SZE WEI

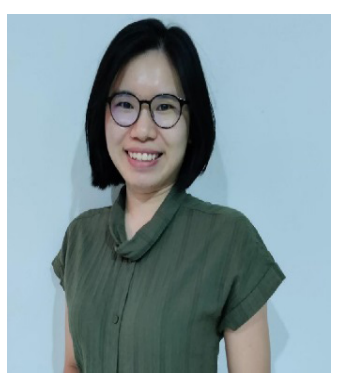

CHANG XIN NI

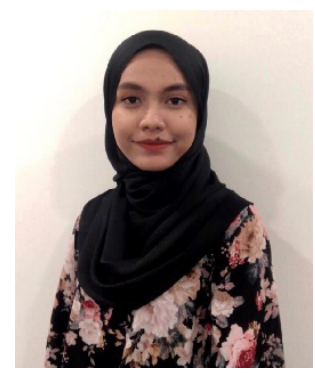

IFFAH ADLINA IBRAHIM

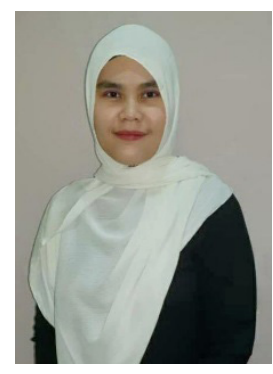

NURUL ASYIKIN ZAINAL ABIDIN

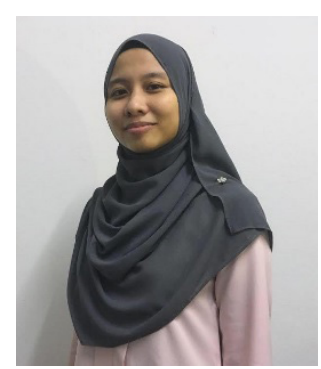

HANISAH MUHAMMAD FAIZ

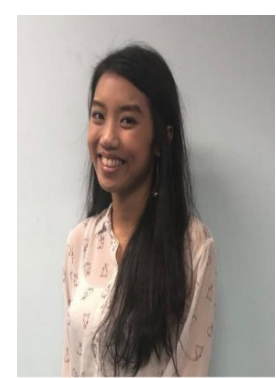

CHIANG YING JEE

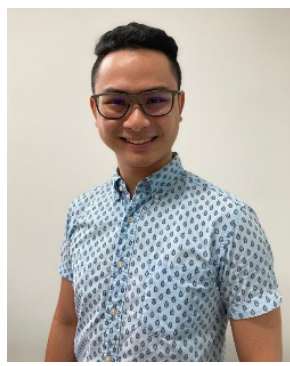

IZMIR ARMANI ISTAL ZANI

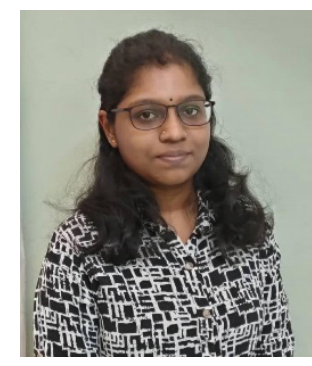

DHIVIYA GOPAL BALAKRISHNAN

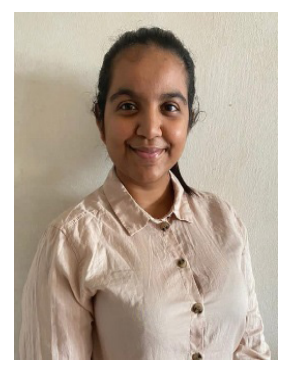

KAVISHASHREE VIJAYAKUMAR

Figure 5• (continued on next page) 
Editorial | Neurotechnology STIE and human resources Malaysia

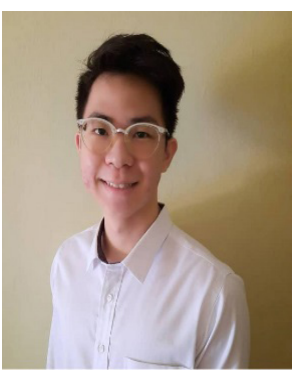

LIM ROONZOE

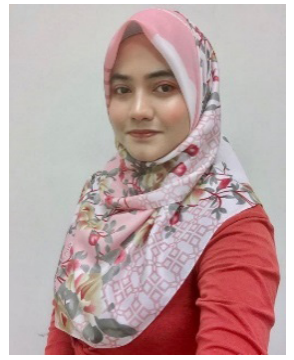

NOR SYALIZA AHMAD

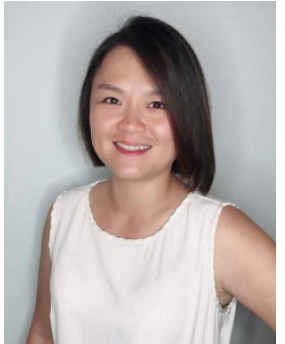

ONG BOON HEE

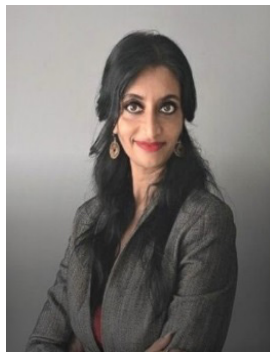

SUBASHINY KALIAPERUMAL

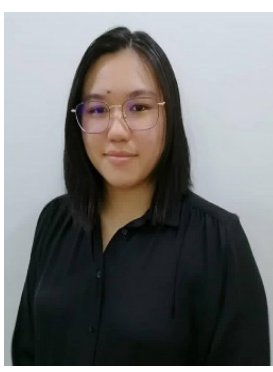

MELISSA LIM ZI QI

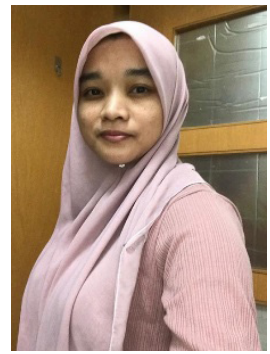

NUR AKHTAR AB RASAB

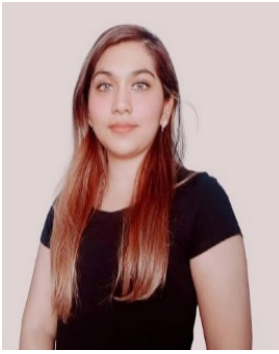

SALINI MANIMARAN

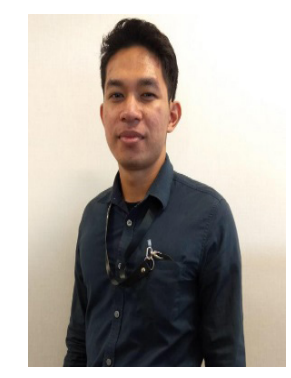

MOHAMAD FIRDAUS CHE FAUZI

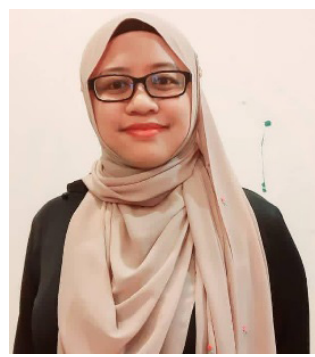

NUR AYUNIE AYOB

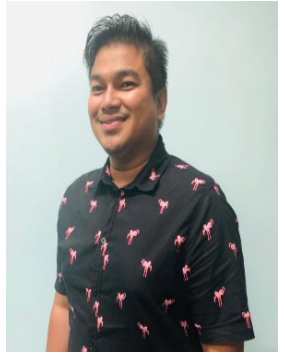

MOHD AZMARUL A AZIZ

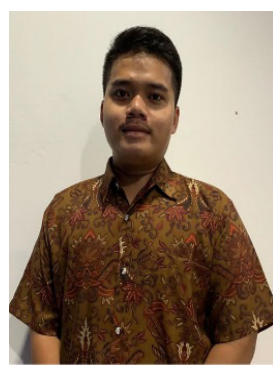

MUHAMMAD

SYAMIL

KAMARUDIN

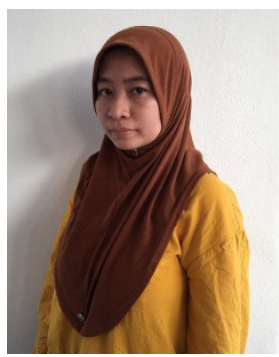

NUR MADIHAH AZMAN

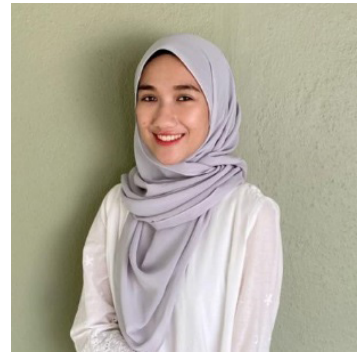

SITI SYAHMINA SHUHAINOR

Figure 5. Third, fourth and fifth batch of Masters of Cognitive Neurosciences USM offered at Postgraduate Institute @Kuala Lumpur 
The Malaysian Brain Mapping project that uses various neurotechnologies (electroencephalography, functional magnetic resonance imaging, event related potential, eye tracking, magnetoencephalography, deep brain microrecording, near infrared spectroscopy) has risen from its humble beginnings as an initiative of the Universiti Sains Malaysia Brain Mapping Group in 2017. There is currently a proposal for its entry into the national arena via the Precision Medicine Initiative with the Academy Science Malaysia, the Ministry of Science, Technology and Innovation, and the Ministry of Health in a recent meeting with the Academy of Science Malaysia in early 2021. This is a Malaysia's parallel initiative of the successful Cuban Brain Mapping Project which was published recently (8).

Thus, Malaysia must have a Centre of Excellence for Clinical Neuroscience, Psychiatry and Psychology services that, at least, represents the cluster of hospitals and teaching institutions with clinical neurosciences as well as psychiatry and clinical psychological services situated in the east coast of West Malaysia in the 12th Malaysia Plan that emphasises the use of neurotechnology in healthcare. It is also important to consistently build the younger generation of neuroscientists, neurologists, neurosurgeons, neurorehabilitation specialists, clinical psychologists and clinical neuropsychologists since it takes nearly 11 to 16 years to train them to address the needs of the country using neurotechnology to diagnose and cure diseases.

\section{Correspondence}

Professor Dato' Dr Jafri Malin Abdullah MD (USM), PhD (University of Ghent, Belgium), FRCS (Ed), FACS (USA), DSCN (Belgium), FAMM, FRSM (UK), FICS (USA)

i) Brain and Behaviour Cluster, School of Medical Sciences, Universiti Sains Malaysia, 16150 Kubang Kerian, Kelantan, Malaysia.

ii) Department of Neurosciences, School of Medical Sciences, Universiti Sains Malaysia, 16150 Kubang Kerian, Kelantan, Malaysia.

iii) Department of Neurosciences, Hospital Universiti Sains Malaysia, Universiti Sains Malaysia, 16150 Kubang Kerian, Kelantan, Malaysia.

Tel: +6097676300

Fax: +609 7673833

E-mail: brainsciences@gmail.com

\section{References}

1. Bernama News. Strengthen STI Mastery for socioeconomic progress-PM Mahyuddin Yassin. 2020 July 14. Available at: https://www.pmo.gov. my/2020/o7/strengthen-sti-mastery-for-socioeconomic-progress-pm-muhyiddin/

2. Academy of Sciences Malaysia. 10-10 Malaysian Science, Technology, Innovation and Economy (MySTIE) framework. Trailblazing the way for prosperity, societal well-being \& global competitiveness; 2020. Available at: http://www. akademisains.gov.my/10-10-mystie/

3. MySTIE $10-10$ - An integrated policy to revolutionize S\&T. The petri dish; 2020 Dec 30. Available at: https://thepetridish. my/2020/12/30/mystie-10-10-an-integratedpolicy-to-revolutionize-st/

4. Establishment of RM1 billion High Tech Facility-National Investment Aspirations (HTFNIA). Announcement Bank Negara Malaysia 15th Dec 2020. https://www.bnm.gov.my/-/ establishment-of-rm1-billion-high-tech-facilitynational-investment-aspirations-htf-nia-1

5. Abdullah JM. Neurosciences at Universiti Sains Malaysia represent Malaysia to support global precision brain health. Malays $J$ Med Sci. 2019;26(3):1-23. https://doi.org/10.21315/ mjms2019.26.3.1

6. Shekh Ibrahim SA, Hamzah N, Abdul Wahab AR, Abdullah JM, Ahamed Hassain Malim NH, Sumari P, et al. Big data initiative in Universiti Sains Malaysia: challenges in brain mapping for Malaysia. Malays J Med Sci. 2020;27(4):1-8. https://doi.org/10.21315/mjms2020.27.4.1

7. https://www.topuniversities.com/universityrankings/employability-rankings/2020

8. Valdes-Sosa PA, Galan-Garcia L, Bosch-Bayard J, Bringas-Vega ML, Aubert-Vazquez E, RodrigeuzGil I, et al. The Cuban Human Brain Mapping Project, a young and middle age population-based EEG, MRI and cognition dataset. Scientific Data. 2021;8:45. https://doi.org/10.1038/s41597-02100829-7 